\title{
Unraveling the Temporal Dynamics of Reward Signals in Music-Induced Pleasure with TMS
}

\author{
Ernest Mas-Herrero, ${ }^{1,2}$ Alain Dagher, ${ }^{3}{ }^{\circledR}$ Marcel Farrés-Franch, ${ }^{3,4}$ and Robert J. Zatorre ${ }^{3,4,5}$ \\ ${ }^{1}$ Cognition and Brain Plasticity Unit, Bellvitge Biomedical Research Institute, L'Hospitalet de Llobregat, 08907 Barcelona, Spain, ${ }^{2}$ Department of \\ Cognition, Development and Education Psychology, University of Barcelona, 08035 Barcelona, Spain, ${ }^{3}$ Montreal Neurological Institute, McGill \\ University, Montreal, Quebec H3A 2B4, Canada, ${ }^{4}$ International Laboratory for Brain, Music and Sound Research,Montreal, Quebec H2V 2J2, \\ Canada, and ${ }^{5}$ Centre for Interdisciplinary Research in Music Media and Technology, Montreal, Quebec H3A 1E3, Canada
}

Music's ability to induce feelings of pleasure has been the subject of intense neuroscientific research lately. Prior neuroimaging studies have shown that music-induced pleasure engages cortico-striatal circuits related to the anticipation and receipt of biologically relevant rewards/incentives, but these reports are necessarily correlational. Here, we studied both the causal role of this circuitry and its temporal dynamics by applying transcranial magnetic stimulation (TMS) over the left dorsolateral PFC combined with fMRI in 17 male and female participants. Behaviorally, we found that, in accord with previous findings, excitation of fronto-striatal pathways enhanced subjective reports of music-induced pleasure and motivation, whereas inhibition of the same circuitry led to the reduction of both. fMRI activity patterns indicated that these behavioral changes were driven by bidirectional TMS-induced alteration of fronto-striatal function. Specifically, changes in activity in the NAcc predicted modulation of both hedonic and motivational responses, with a dissociation between pre-experiential versus experiential components of musical reward. In addition, TMS-induced changes in the fMRI functional connectivity between the NAcc and frontal and auditory cortices predicted the degree of modulation of hedonic responses. These results indicate that the engagement of cortico-striatal pathways and the NAcc, in particular, is indispensable to experience rewarding feelings from music.

Key words: motivation; music; NAcc; pleasure; reward; TMS

Significance Statement

Neuroimaging studies have shown that music-induced pleasure engages cortico-striatal circuits involved in the processing of biologically relevant rewards. Yet, these reports are necessarily correlational. Here, we studied both the causal role of this circuitry and its temporal dynamics by combining brain stimulation over the frontal cortex with functional imaging. Behaviorally, we found that excitation and inhibition of fronto-striatal pathways enhanced and disrupted, respectively, subjective reports of music-induced pleasure and motivation. These changes were associated with changes in NAcc activity and NAcc coupling with frontal and auditory cortices, dissociating between pre-experimental versus experiential components of musical reward. These results indicate that the engagement of cortico-striatal pathways, and the NAcc in particular, is indispensable to experience rewarding feeling from music.

Received Mar. 30, 2020; revised Dec. 18, 2020; accepted Dec. 21, 2020.

Author contributions: E.M.-H., A.D., and R.J.Z. designed research; E.M.-H. and M.F.-F. performed research; E.M.-H. analyzed data; E.M.-H. wrote the first draft of the paper; E.M.-H., A.D., and R.J.Z. edited the paper; E. M.-H. wrote the paper.

R.J.Z. is supported a Foundation Grant from the Canadian Institutes of Health Research, a Discovery Grant from the Natural Sciences and Engineering Research Council of Canada, and the Canada Fund for Innovation. R.J.Z. is a fellow of the Canadian Institute for Advanced Research. A.D is funded by the CIHR Foundation Grant. E.M.-H. is supported by a fellowship from "la Caixa" Foundation (ID: 100010434, fellowship code: LCF/

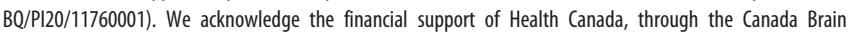
Research Fund in partnership with the Montreal Neurological Institute, and of the Healthy Brains for Healthy Lives initiative of McGill University. The funders had no role in the conceptualization, design, data collection, analysis, decision to publish, or preparation of the manuscript.

The authors declare no competing financial interests.

Correspondence should be addressed to Ernest Mas-Herrero at emasherrero@ub.edu or Robert J. Zatorre at robert.zatorre@mcgill.ca.

https://doi.org/10.1523/JNEUROSC1.0727-20.2020

Copyright $\odot 2021$ the authors

\section{Introduction}

Music can act as a powerful motivational force in our everyday life, driving us toward music-related activities at the expense of time, money, and effort: from waiting in line for hours in the rain or snow to buy a concert ticket to investing years of training to play an instrument. Neuroimaging studies have shown that, despite the sophistication, complexity, and abstractness of music perception, music-induced pleasure relies on an otherwise evolutionary ancient circuitry: the so-called reward circuit (Blood and Zatorre, 2001; Koelsch et al., 2006; Salimpoor et al., 2011, 2013; Martínez-Molina et al., 2016; Brattico et al., 2016). This circuit comprises both striatal (NAcc, caudate, and putamen) and cortical regions [the ventromedial PFC (vmPFC)], constituting a complex network that is known to be involved in different 

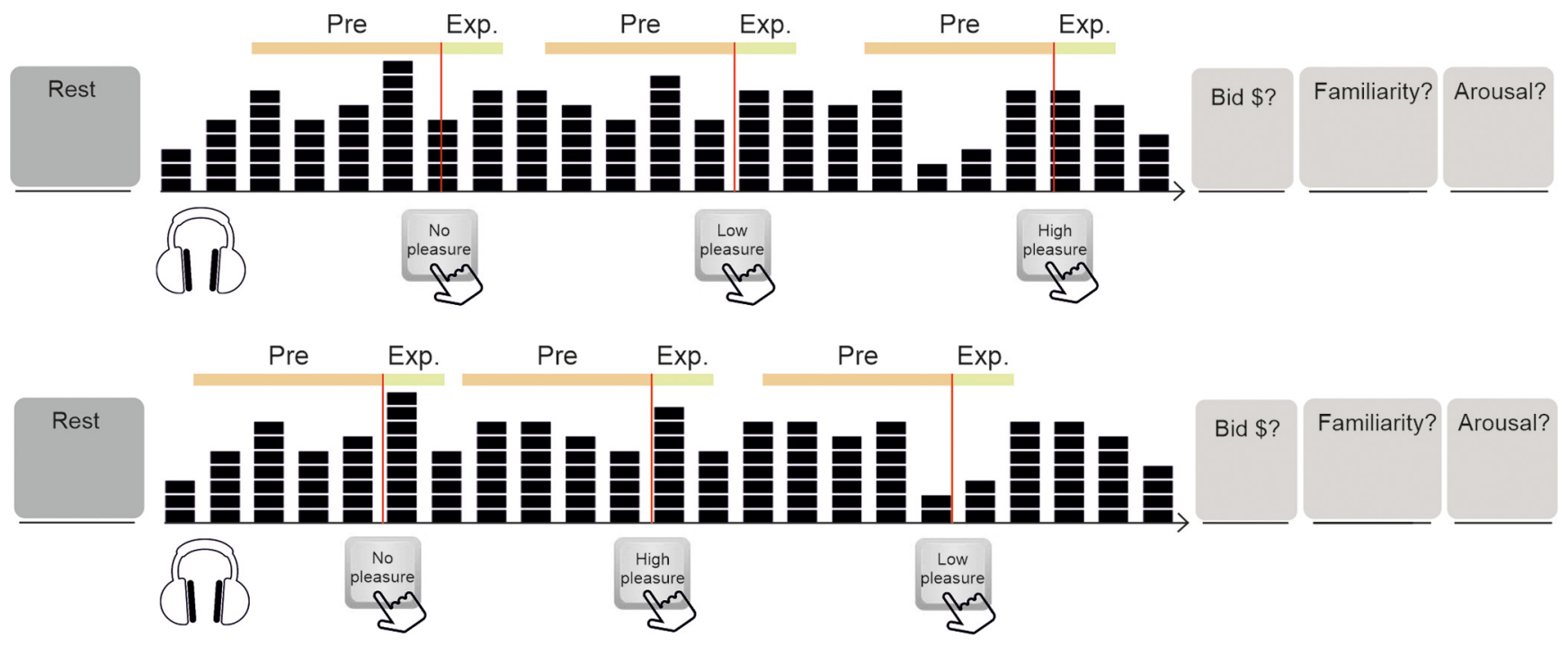

Figure 1. Schematic of the experimental paradigm. Each trial started with a fixation cross lasting $20 \mathrm{~s}$, followed by a musical excerpt (with a duration of $45 \mathrm{~s}$, in the figure represented by the power spectral density of an audio track). While listening to music, the participants had to rate the degree of pleasure they were experiencing in real time by pressing the corresponding button. Real-time ratings of pleasure were used to identify Pre-experience and Experience time periods. The Experience phase was modeled as events time-locked to the moment at which a participant pressed a button to indicate a change in pleasure ratings. The green line is only for illustrative purposes since durations were set to 0 (see Materials and Methods). The Pre-experience epochs were defined as the $10 \mathrm{~s}$ before a button press. At the end of each excerpt, participants had to indicate the amount of money they were willing to pay (only for the experimenter-selected excerpts), the familiarity, and the arousal. Pre, Pre-experience; Exp., Experience.

aspects of learning and motivation in response to reward and incentive salience signals (Bartra et al., 2013; Sescousse et al., 2013).

Evidence from research on primary and secondary rewards indicates that this circuitry, guided by dopaminergic signaling from the midbrain, responds in at least two distinct temporal phases within the reward cycle: before and after the eventual reward is received (Schultz et al., 1997; Luijten et al., 2017). In both cases, activation of striatal, vmPFC, and dopaminergic neurons has been related to reward-related signals, such as expected value, motivation, incentive salience, and reward prediction errors (Bromberg-Martin et al., 2010; Chase et al., 2015; MasHerrero et al., 2019; Diekhof et al., 2012). Analogously, Salimpoor et al. (2011) showed that dopamine release occurs at the anticipation and the peak experience of musical chills, in the caudate and the NAcc, respectively. Notably, dopamine release in both striatal regions was correlated with hedonic reactions to music

However, neuroimaging methods are correlational in nature; and thus, while they may reflect true causal mechanisms, correlational activities may not distinguish between brain regions directly involved in generating the hedonic experience from those that are only modulated by this experience. We have recently bridged this gap by using transcranial magnetic stimulation (TMS) over the left dorsolateral PFC (dlPFC) (Mas-Herrero et al., 2018a), a procedure previously shown to effectively and noninvasively induce dopamine release and BOLD activations in the striatum (Strafella et al., 2001; Hayashi et al., 2013). By applying TMS with excitatory and inhibitory stimulation protocols, we were able to upregulate and downregulate behavioral and psychophysiological measures of musical pleasure and motivation to purchase music (Mas-Herrero et al., 2018a). Relatedly, pharmacological manipulation of dopaminergic activity has also been shown to modulate musical pleasure and motivation bidirectionally (Ferreri et al., 2019). These types of manipulations provide clear evidence for a causal role of striatal dopamine in musical pleasure but do not reveal the temporal dynamics of fronto-striatal signals or the neural substrates of music-induced pleasure.

Here, by combining TMS over the left dlPFC with fMRI, we aimed to provide a deeper understanding of the fronto-striatal circuitry's role in music reward. As in our previous behavioral study, participants were tested in three separate sessions (at least $24 \mathrm{~h}$ apart) in which either excitatory (intermittent theta burst stimulation [iTBS]), inhibitory (continuous theta burst stimulation [cTBS]), and Sham stimulation was applied in a counterbalanced fashion. Immediately following the stimulation, participants entered into the MRI scanner where they performed the same musical paradigm that we previously developed (Mas-Herrero et al., 2018a; Ferreri et al., 2019). The participants listened to self-selected favorite and experimenter-selected musical clips while providing continuous real-time ratings of experienced pleasure (Fig. 1). In addition, they had the opportunity to purchase our music selections using an auction paradigm (Salimpoor et al., 2013). We hypothesized that, if the functioning of the fronto-striatal circuitry underlies the TMS-induced changes in musical pleasure and motivation, then: (1) iTBS and cTBS should result in increases and decreases, respectively, of the engagement of this circuitry, as measured by task-related BOLD activity and functional connectivity; (2) and, in turn, subject-specific TMS-induced changes in the functioning of these regions should predict changes in subjective reports of pleasure and/ or motivation to purchase music across participants.

\section{Materials and Methods}

Participants. Eighteen right-handed participants (11 females, mean $=24.3$ years, $\mathrm{SD}=4.2$ years) with no formal musical training were recruited. Participants had no history of neurologic disease or hearing impairment. A screening question was asked before the study to ensure that all participants preferred pop music since that was the music genre selected for the experiment. All participants gave their informed consent, and the protocol was approved by the Montreal Neurologic Institute Ethics Review Board. Participants were informed that the goal of the 
study was to determine the role of reward circuits in music-induced emotion and motivation, but they were not informed about the specific hypothesis nor the difference among different stimulation sessions. One participant did not complete one of the sessions and, thus, was excluded from the analysis. The sample size was chosen based on a previous study showing modulation of music reward-related responses following a similar TMS design than that used in the current study (Mas-Herrero et al., 2018a).

Music task. Each session consisted of one run in which the participants listened to 5 self-selected songs and 10 experimenter-selected songs (songs were selected following the same procedure as in MasHerrero et al., 2018a). The order of presentation of both groups of songs was counterbalanced across participants. The order of presentation of songs was fully randomized. The participants had to indicate, in realtime, their degree of pleasure while listening to the music by pressing one of four different buttons on an MRI-compatible response pad $(1=$ neutral, $2=$ low pleasure, $3=$ high pleasure, $4=$ chill $)$. The participants were instructed to hold down the button as long as they experienced the corresponding degree of pleasure. At the end of each excerpt, the participants were asked to rate the familiarity (from $1=$ unfamiliar to $4=\mathrm{I}$ have the song on my PC, mp3, Spotify list, etc.) and arousal (from $1=$ not at all arousing to $4=$ highly arousing) they felt in response to the musical excerpt. The songs from the experimenter selection that the participants reported to own were discarded from the analysis (mean $=0.74$, $\mathrm{SD}=0.09$ ). In addition, the participants had the opportunity to purchase the experimenter-selected music (not their favorite songs) with their own money in an auction paradigm following the same procedure as described previously (Salimpoor et al., 2013; Mas-Herrero et al., 2018a; Ferreri et al., 2019). Participants were instructed to keep their eyes open, but no visual feedback was presented while listening to music.

Experimental design. Each participant performed three fMRI sessions in which different transcranial magnetic stimulations were applied (iTBS, cTBS, or Sham) over the left dlPFC. The left dlPFC was chosen as a target based on previous evidence indicating that dopamine release and BOLD activity in reward-related structures (striatum and vmPFC) may be modulated by applying TMS over this region (Strafella et al., 2001; Hayashi et al., 2013) but not over the right dlPFC (see Cho and Strafella, 2009). Concretely, the coordinates selected for the left dlPFC ( $x=-40, y=32$, and $z=30$ ) were based on Strafella et al. (2001), which showed striatal dopamine release following excitatory TMS stimulation over this coordinate. In order to localize the target coordinate, we used T1-weighted high-resolution MRI from each participant. The Talairach coordinates were converted into MNI coordinates and then into the subject's native MNI space using the reverse native-to-MNI transformation from SPM. A real-time optically tracked frameless stereotaxic system (Brainsight Frameless, Rogue Research) was used to guide the coil over the subject's scalp. An infrared camera for online subject tracking and coil positioning (Polaris Spectra, NDI) was used.

The coil was held in a fixed position by a mechanical arm (which provided flexible positioning and rotation of the coil in multiple directions) over the target area. It was oriented so that the induced electric current flowed in a posterior-anterior direction. The stimulation took place in a room next to the MRI suite. TMS was applied using a Magstim Super Rapid stimulator. Stimulation intensity was set to $40 \%$ of the maximum stimulator output, following the protocol of our previous study (Mas-Herrero et al., 2018a). The Sham stimulation was delivered with the coil positioned at a perpendicular angle to the skull area using either the iTBS or the cTBS protocol, in a counterbalanced manner across participants. Immediately after the stimulation, participants were positioned into the MRI camera. Then, they performed the music listening task, which lasted $\sim 20 \mathrm{~min}$. Next, a high-resolution structural image was acquired. Stimulation conditions were counterbalanced across participants. There was at least a $24 \mathrm{~h}$ interval between sessions to minimize potential carryover effects.

fMRI data acquisition. fMRI data were collected using a Siemens TIM Trio 3T scanner and a 32-channel head coil at the McConnell Brain Imaging Center of the MNI. Functional images sensitive to BOLD contrast were acquired using an echo-planar $\mathrm{T} 2{ }^{*}$ weighted gradient echo sequence $\left(38\right.$ slices, $\mathrm{TR}=2300 \mathrm{~ms}, \mathrm{TE}=30 \mathrm{~ms}$, flip angle $90^{\circ}, 3.5 \mathrm{~mm}$ isotropic voxels). High-resolution T1-weighted images (MPRAGE: $\mathrm{TE}=2.98 \mathrm{~ms}, \mathrm{TR}=2300 \mathrm{~ms}$, matrix size $=64 \times 64 \times 192,1 \mathrm{~mm}$ isotropic voxels) were acquired immediately after the functional images. To reduce susceptibility artifacts in the orbitofrontal cortex and the anterior parts of the ventral striatum, slices were orientated with an angle of 30 degrees with the plane intersecting the anterior and the posterior commissures (Weiskopf et al., 2006). The data are available from the corresponding author on request.

Statistical analysis. The reward system's intrinsic functioning is reflected by the values of the dependent variables measured in the Sham condition when no brain modulation occurred. In this study, cTBS and iTBS were chosen as the means to "displace" this intrinsic state in opposite directions. Therefore, here we aimed to investigate whether modulation of the reward system by means of TMS influenced the variables under study (i.e., liking and wanting), rather than assessing the capacity of the cTBS and iTBS protocols themselves to block or enhance, respectively, the intrinsic reward-related responses. For that reason, our analyses focused on comparing the cTBS and iTBS data against each other by using the Sham session as a baseline. This procedure also controls for variance associated with individual differences by providing a baseline correction.

First, we aimed to replicate our previous behavioral findings (MasHerrero et al., 2018a). To investigate the effect of both cTBS (inhibitory protocol) and iTBS (excitatory protocol) over the left dlPFC on experienced pleasure, we computed a "liking rate" for each song based on participants' ratings while listening to the music. The liking rate was computed by multiplying the response value, -1 (no pleasure), 2 (low pleasure), 3 (high pleasure), or 4 (chill), by the duration of each response and divided by the total duration of the song. In other words, we computed a weighted average of the ratings. Then the resulting "liking rates" were averaged for each session. Next, we computed percentage of change with respect to the Sham session for both the iTBS and cTBS sessions for each participant. To explore the effect on both self-selected and experimenter-selected stimuli, we computed changes separately for each group of songs. We then performed a repeated-measures ANOVA with musical clip and session as within-subject factors. On average, participants reported a similar number of ratings on each session (iTBS $=43.7$ ratings, Sham $=43.5, \mathrm{cTBS}=43.4, F<1$ ). Following iTBS, participants reported "no pleasure" in $22.18 \%$ of the trials (among the total amount of ratings reported), "low pleasure" in $37.3 \%$, "high pleasure" in $33.14 \%$, and "chills" in $7.38 \%$. Following Sham, participants reported "no pleasure" in $21.96 \%$ of the occasions, "low pleasure" in $39.10 \%$, "high pleasure" in $33.79 \%$, and "chills" in $5.17 \%$. Finally, following cTBS, participants reported "no pleasure" in $25.73 \%$ of the occasions, "low pleasure" in $36.77 \%$, "high pleasure" in $32.62 \%$, and "chills" in $4.87 \%$.

We also aimed to investigate the effect of cTBS and iTBS over the left dlPFC on the motivation to listen to music. To study changes in motivation, we analyzed the amount of money participants were willing to pay to purchase the music heard in each session, using a similar approach to that of Salimpoor et al. (2013). We computed percentage of change with respect to the Sham condition and performed a one-tailed paired-sample $t$ test between percentage change following iTBS and cTBS.

Finally, we also tested differences across stimulation sessions in the number of reported chills and their time duration. Sham-corrected values were compared between active stimulations using a two-tailed paired-sample $t$ test.

fMRI data analysis. Data were preprocessed using Statistical Parametric Mapping software (SPM8; Wellcome Trust Center for Neuroimaging, University College London). Functional runs were first slice timing-corrected and realigned. Then, the bias-corrected structural image was coregistered to the mean functional image and segmented by means of the Unified Segmentation implemented in SPM8. The resulting normalization parameters were applied to all functional images. Finally, functional images were spatially smoothed with an $8 \mathrm{~mm}$ FWHM kernel.

The resulting fMRI time series were analyzed at the first (subject), level using one GLM, including all three sessions for each participant and reward phase (Pre-experience and Experience). Nine task-related regressors were included in each model. Experimenter and self-selected 
a

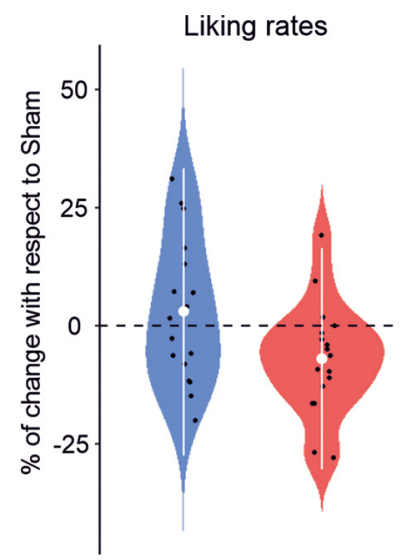

b

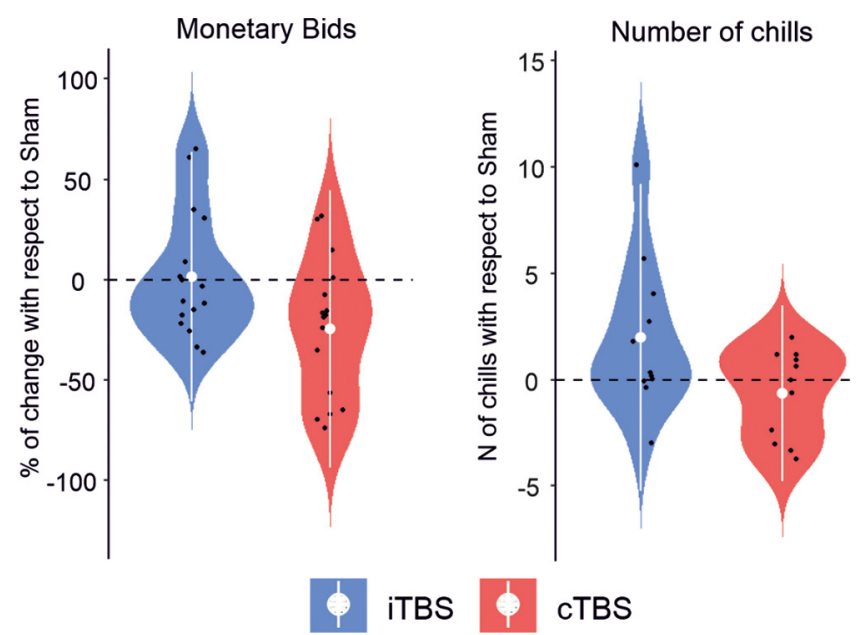

d

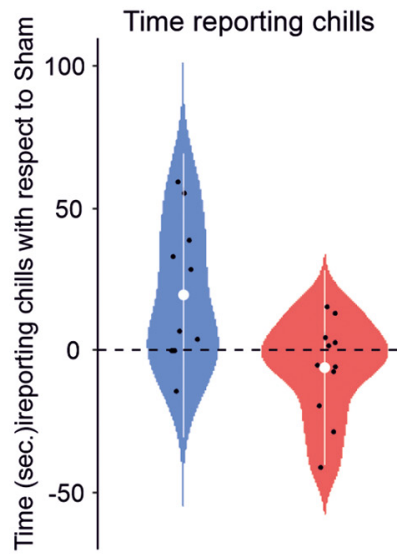

Figure 2. Main behavioral results of the study. Change with respect to the Sham condition following both iTBS and cTBS for liking rates (illustrating the main effect of TMS session from the repeated-measures ANOVA, averaged across self- and experimenter-selected excerpts, $d=0.81)(\boldsymbol{a})$, the money spent to buy experimenter-selected music $(d=0.50)(\boldsymbol{b})$, and the number $(d=0.51)(c)$ and time $(d=0.57)(d)$ reporting chills. In all four variables, the excitatory iTBS condition leads to positive change, whereas the inhibitory cTBS condition has the opposite effect. Violin plots represent kernel probability density. White circle dots represent the average. White error bars indicate SD.

excerpts were modeled time-locked to the end of the fade-in, that is, $5 \mathrm{~s}$ after the onset of each excerpt for a $40 \mathrm{~s}$ duration. Separate regressors to model the first $5 \mathrm{~s}$ of each excerpt, the presentation of ratings regarding arousal, familiarity, and wanting at the end of each excerpt (duration $=0$ ), and a regressor with the ratings provided in these post-song judgments (duration $=0$ ) were also specified in the design matrix. The "rest" condition was modeled in a separate regressor with a $20 \mathrm{~s}$ duration. Finally, real-time pleasure ratings were used to identify Pre-experience and Experience time periods. As in MartínezMolina et al. (2016) and Salimpoor et al. (2011), the Experience phase was modeled as events time-locked to the moment at which a participant pressed a button to indicate a change in pleasure ratings (e.g., at the time a participant suddenly report to experience greater pleasure by pressing button 3 o 4; duration=0). In addition, we were also interested in the time window just before the button was pressed, that is, the Pre-experience period. Our motivation to look at this phase comes from (1) the identification of anticipatory-related responses to chills in abstract rewards (Salimpoor et al., 2011; Wassiliwizky et al., 2017); (2) theoretical models holding that musical pleasure is buildup through time, and highly dependent on the preceding context (Meyer, 1956; Sloboda, 1991; Huron, 2006); and (3) reinforcement learning models of reward processing, showing that expected value of upcoming rewards, encoded in reward-related structures, such as the striatum, frequently fluctuates as events unfold over time, by either increasing or decreasing the value of what it is about to come, given the current circumstances (Schultz et al., 1997; Mas-Herrero et al., 2019). The few studies that have explored this phase using abstract rewards have specifically looked at the anticipation of chills and have generally treated this period as a sustained response lasting for a few seconds before the experience of chills (from 6 to $15 \mathrm{~s}$ before pressing a button reflecting the occurrence of a chill) (Salimpoor et al., 2011; Wassiliwizky et al., 2017). Thereby, we defined Pre-experience epochs as the $10 \mathrm{~s}$ before a button press conforming to the average duration spent at a particular rating level in the current experiment (mean $=14.94 \mathrm{~s}, \mathrm{SD}=4.64 \mathrm{~s}$ ). Therefore, the Pre-experience epoch of one rating and the Experience of the previous were unlikely to overlap. Those trials in which the Pre-experience overlapped with the previous rating epoch were excluded from the analysis (mean $=8.00$ ratings/session; $\mathrm{SD}=4.5$ ). For both Pre-experience and Experience, a first-order parametric regressor modeled the pleasure rate (range: 14). Finally, 24 motion regressors were also included to account for movement-related variance. All regressors were subsequently convolved with the canonical HRF.
Given our explicit a priori hypothesis regarding the striatum and the vmPFC, an ROI analysis was performed, including the left and right NAcc, the left and right caudate, the left and right putamen, and the left and right vmPFC. Striatal ROIs were created based on anatomic masks from the probabilistic atlas of Hammers et al. (2003). The vmPFC ROI was created based on a functional cluster from a previous meta-analysis on subjective hedonic value (SHV) (Bartra et al., 2013). To control our findings' specificity, we also performed an ROI analysis over the primary visual cortex, which was created in the left and right calcarine cortex according to predefined anatomic masks (AAL database). Finally, an ROI in the dlPFC was defined by drawing a $10 \mathrm{~mm}$ sphere around the peak coordinates of the stimulation target.

First, we aimed to confirm that the main effect of subjective value was present on each session during the Pre-experience and Experience phases in the circuitry formed by striatal regions and the vmPFC, as previous studies on reward processing have shown (Bartra et al., 2013; Oldham et al., 2018). With this purpose in mind, the main contrasts of interest (SHV contrast), testing the slopes of SHV regressors, were built at the first (subject) level for the Pre-experience (reflecting value expectancy) and the Experience phases (reflecting the pleasure experienced). For each participant, we averaged the $\beta$ coefficients within all the reward-related ROIs (averaging bilateral NAcc, caudate, putamen, and vmPFC) for each stimulation session. We tested whether the group average estimates were significantly different from zero using one-tailed $t$ tests.

In order to explore differences between the two active sessions in the SHV, the Sham session was used as a baseline and subtracted from both iTBS and cTBS sessions at the first (subject) level, leading to four contrast: changes following iTBS and cTBS during both the Pre-experience and the Experience phases as a function of subjective value. Individual mean $\beta$ coefficients were then extracted from the subjects' first level fMRI analysis for each ROI and entered in a $2 \times 4 \times 2 \times 2$ repeatedmeasures ANOVA with the factors stimulation session (iTBS, cTBS), ROI (NAcc, caudate, putamen, and vmPFC), hemisphere (left, right), and reward phase (Pre-experience, Experience). For the correlational analysis, differences between iTBS and cTBS were computed by subtracting changes following cTBS from changes following iTBS for each ROI and phase in SHV. Correlational analyses were run using robust-fit regression to reduce the influence of any potential outlier. To account for multiple comparisons, Bonferroni corrections were applied as a function of the number of regions analyzed on each contrast $(n=8)$; thus, significant $p$ values were set to $0.05 / 8=0.00625$. To compare between correlation coefficients, we followed the procedure formulated by Steiger (1980) in a one-sided asymptotic $z$ test. 


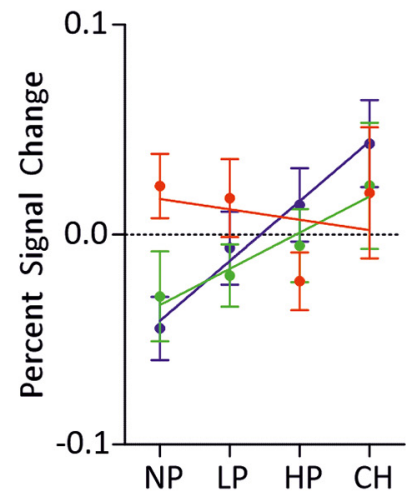

b

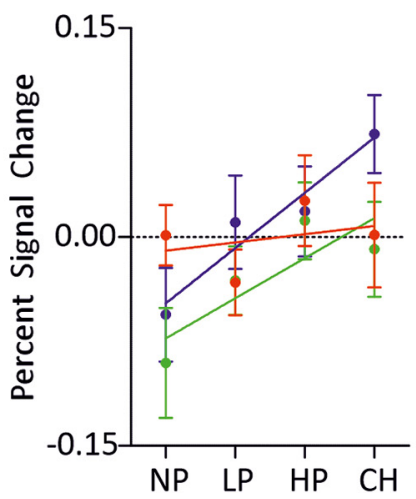

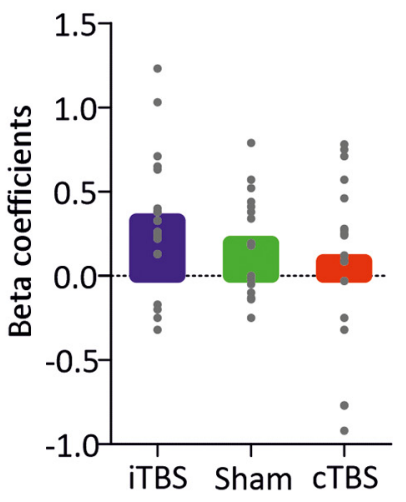

Experience

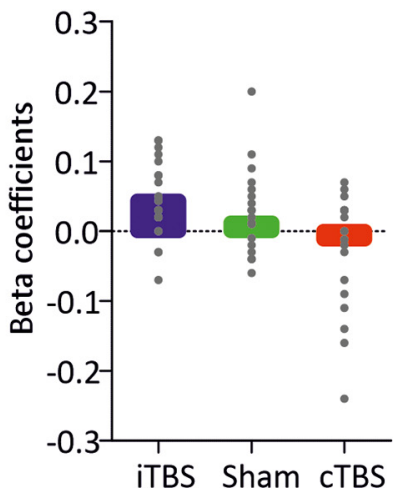

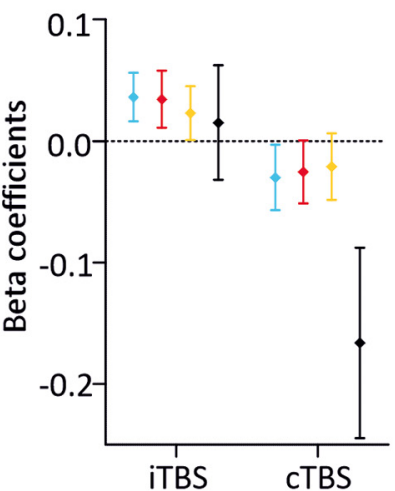

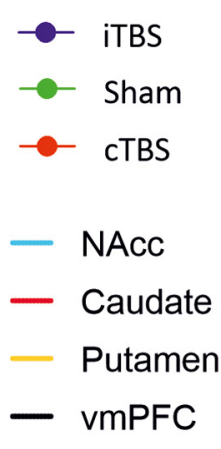

C

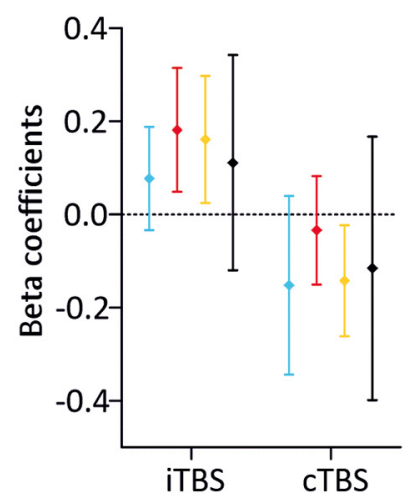

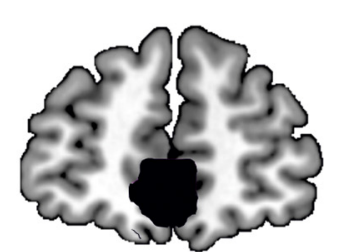

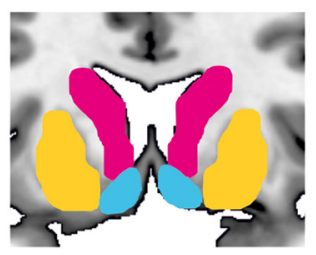

Figure 3. Main fMRI results. $\boldsymbol{a}, \boldsymbol{b}$, Left, Graph represents the parametric effect of SHVs (top, Pre-experience; bottom, Experience) on percent signal change for the reward circuitry (including bilateral NAcc, caudate, putamen, and vmPFC). Note the linear increase in percent signal change as the pleasure increase during both the Pre-experience and the Experience following iTBS and Sham sessions, but not cTBS. Middle, Bar graph represents the resulting slopes (individual and the average) of the SHV regressor for each session and phase. Right, Contrast estimates reflecting the slopes of the SHVs regressors following either iTBS or cTBS with respect to Sham for each of the ROls and reward phase. C, Reward-related ROIs included in the study. NP, No pleasure; LP, low pleasure; $\mathrm{HP}$, high pleasure; $\mathrm{CH}$, chills.

Interregional functional connectivity analysis. We used a psychophysiological interaction (PPI) (Friston et al., 1997) analysis to assess whether connectivity changes between the left dlPFC or the superior temporal gyrus (STG) to the rest of the musical reward circuitry were predictive of TMS-induced changes of pleasure and motivation. Seed ROIs were defined individually around the single subject peak value ( $5 \mathrm{~mm}$ radius spheres) of each contrast (hedonic value during both the Pre-experience and the Experience) during the Sham session within the left dIPFC and the left and right STG. STG was defined using the probabilistic neuroanatomical adult atlas developed by Hammers et al. (2003), merging the anterior and posterior parts of the STG to generate one mask for each hemisphere (as in Martínez-Molina et al., 2016). For all participants, individual deconvolved time-series were extracted from all voxels within these spheres. The elementby-element product of the extracted time-series (the first eigenvariate from every voxel in the sphere) and a vector that coded the main effect of task were then calculated. The result of this product was then reconvolved with the canonical HRF to create the final PPI regressor. For each individual, three extended GLM models were built (one for the left dIPFC, one for the left STG, and one for the right STG) for each reward phase.

The model included the conditions previously defined for the fMRI analysis, the deconvolved time-series, and the derived PPI as regressors. Individual models were estimated, and main contrasts were generated to test the effects of the PPI regressors. Next, we correlated TMS-induced changes in the resulting contrast estimates between iTBS and cTBS (iTBS - cTBS) with the difference in subjective reports of pleasure and motivation using robust-fit regression to reduce the influence of any potential outlier. To account for multiple comparisons, Bonferroni corrections were applied as a function of the number of regions analyzed on each contrast $(n=8$; significant $p$ values were set to $0.05 / 8=0.00625$ )

\section{Results}

\section{Behavior}

We computed a liking rate for each musical excerpt based on participants' real-time ratings obtained during scanning and determined an average for each session. Then, we computed percentage change with respect to the Sham session and performed a repeated-measures ANOVA with selection (self- and experimenter-selected excerpts) and stimulation type (percentage of change following iTBS and cTBS compared with Sham) as withinsubject factors. The analysis revealed a main effect of stimulation type $\left(F_{(1,16)}=7.85, p=0.01\right)$. The main effect of self- versus experimenter-selected-music $\left(F_{(1,16)}=1.15, p=0.30\right)$, and the interaction selection $\times$ stimulation type did not yield significant effects $\left(F_{(1,16)}\right.$ $=2.23, p=0.16$ ). Like our previous findings, TMS stimulation over the left dlPFC modulated SHV regardless of familiarity: iTBS led to a positive increase in self-reports of pleasure, whereas cTBS decreased participants' liking compared with Sham (Fig. 2a) for both self- and experimenter-selected music.

Similar findings were found when we investigated participants' bids to acquire experimenter-selected music as a measure of 
wanting (Fig. 2b). Participants were willing to spend more money following iTBS than CTBS, relative to Sham $\left(t_{(16)}=2.04\right.$, $p=0.029$ ).

We also examined changes in the number and total duration of reported chills during music listening. Bodily reactions, such as "chills," are generally associated with particularly intense and pleasurable responses to music, and they are often used as an indicator of musical pleasure experiences (Grewe et al., 2005, 2009; Salimpoor et al., 2009; Mas-Herrero et al., 2014). TMS stimulation significantly increased the number of chills $\left(t_{(16)}=2.11\right.$, $p=0.05)$ and the time participants spent reporting chills $\left(t_{(16)}=\right.$ $2.35, p=0.03)$ following iTBS compared with cTBS, relative to Sham (Fig. 2c,d). These findings provide an important replication of our previous work showing that TMS over the left dlPFC reliably modulates musical reward sensitivity.

\section{fMRI}

Next, we aimed to explore whether the TMS intervention induced changes in fMRI brain activity related to the Pre-experience and Experience phases of the music pleasure cycle. Given our strong explicit a priori hypothesis regarding the role of the reward circuitry in this process, we performed an ROI analysis, including its main subcortical and cortical structures, that is, the bilateral NAcc, caudate, putamen, and vmPFC. In addition, we included an ROI in the primary visual cortex as a control region and a $10 \mathrm{~mm}$ sphere around the TMS target coordinate in the left dlPFC to assess the specificity of the effects.

While listening to music, participants indicated when they experienced no pleasure, low pleasure, high pleasure, or a chill by pressing a button (each associated with a value from 1 to 4 , respectively); these responses were then used to identify the Preexperience and the Experience phases of music reward (Fig. 1), to differentiate between value expectations and the real pleasure, respectively. The Experience epochs were time-locked to participants' button press, by which they would indicate a change in the experienced pleasure (suddenly experiencing a chill and pressing the number 4 button, for instance; following the same procedure as in Martínez-Molina et al., 2016, 2019). Pre-experience epochs were defined as the $10 \mathrm{~s}$ before the Experience phase (based on previous studies investigating the anticipation of chills in abstract rewards; see Materials and Methods) (Salimpoor et al., 2011; Wassiliwizky et al., 2017).

First, we examined how the activity within our ROIs scaled parametrically with the subjective ratings of pleasure reported with the button press for each of the stimulation sessions and reward phase. The resulting $\beta$ coefficients (SHV contrasts) reflect how steeply SHV scales with BOLD signal within our ROIs, for each condition and stimulation session (Fig. 3a,b). Our main hypothesis is built on a large body of literature showing that the engagement of reward circuitry is positively correlated with SHV during both before (Pre-experience) and after (Experience) reward delivery, reflecting encoding of expected and experienced pleasure, respectively. Thus, to confirm that this positive relationship was present in each session, we extracted and averaged across each of our reward-related ROIs the individual mean $\beta$ coefficients from the SHV contrast for each stimulation session and reward phase. We then tested whether the group average estimates were significantly different from zero using one-sample $t$ tests.

Consistent with previous literature, reported hedonic values positively correlated with the engagement of the circuit (averaging NAcc, caudate, putamen, and vmPFC ROIs) following either iTBS or Sham during both the Pre-experience $\left(\mathrm{SHV}_{\mathrm{iTBS}} t_{(16)}=3.39\right.$,
Pre-Experience

Experience
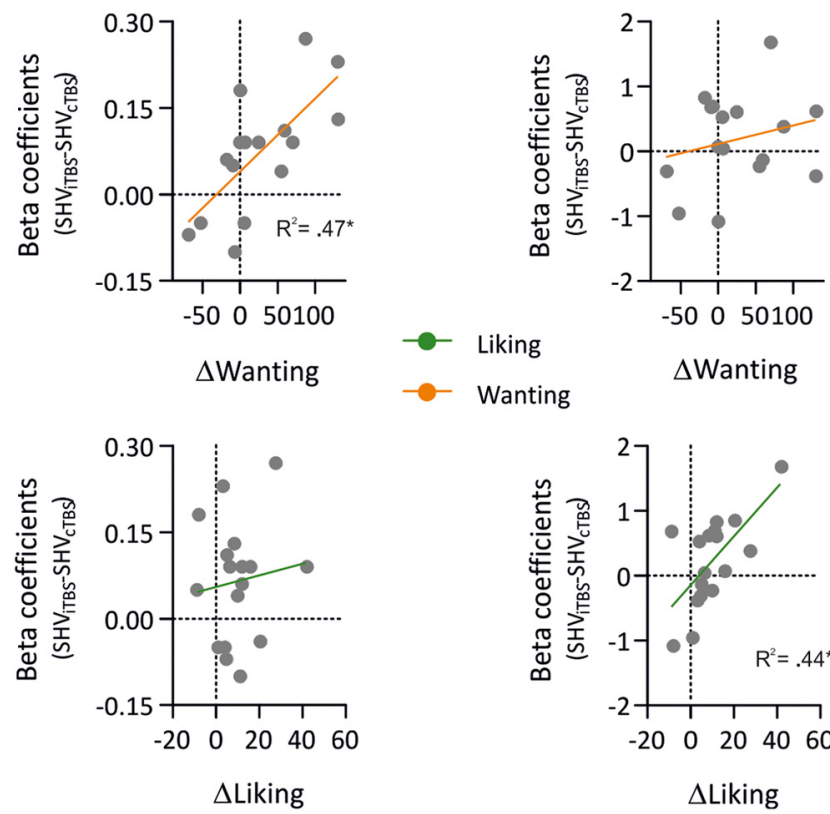

Figure 4. Robust regression analysis. Scatter plots represent the relationship between individual differences in TMS modulation of subjective reports of pleasure and motivation, on the one hand, and subject-specific TMS-induced changes in the NAcc activity during both the Pre-experience and Experience phases. ${ }^{*} P_{\text {bonf }}<0.05$.

$\left.p=0.002 ; \operatorname{SHV}_{\text {Sham }}: t_{(16)}=1.87, p=0.04\right)$ and the Experience phase $\left(\mathrm{SHV}_{\text {iTвS }}: t_{(16)}=3.11, p=0.003\right.$; $\mathrm{SHV}_{\text {Sham }}: t_{(16)}=2.70, p=0.008$; Fig. $3 a, b)$. On the contrary, no correlation was found in either of the two temporal phases following cTBS (Pre-experience: $t_{(16)}=$ $1.44, p=0.09$; Experience: $t_{(16)}=0.66, p=0.26$ ). These initial independent analyses already point to potential differences between the two active stimulation sessions in striatal and vmPFC responses to music reward.

In order to empirically test these differences, and following a similar procedure as in the previous behavioral analysis in which Sham was used as a baseline, we subtracted $\mathrm{SHV}_{\text {Sham }}$ from the SHV contrast of the two active stimulation sessions at the first (subject) level, leading to two main contrast for either the Pre-experience or the Experience phase: changes following iTBS $\left(\mathrm{SHV}_{\mathrm{iTBS}}-\mathrm{SHV}_{\text {Sham }}\right)$ and cTBS $\left(\mathrm{SHV}_{\mathrm{cTBS}}-\mathrm{SHV}_{\text {Sham }}\right)$ with respect to Sham. For each phase and contrast, we extracted the individual $\beta$ coefficients within each of our ROIs, and we then entered them in a $2 \times 4 \times 2 \times 2$ repeated-measures ANOVA with the following within-subject factors: stimulation session (iTBS, cTBS), ROI (NAcc, caudate, putamen, and vmPC), hemisphere (left, right), and reward phase (Pre-experience, Experience). The analysis revealed a main effect of stimulation session $\left(F_{(1,16)}=6.67, p=0.02\right)$ independently of ROI, hemisphere, and reward phase (all $p$ values $>0.20$, including interactions). That is, excitatory stimulation (iTBS) significantly enhanced the responsiveness of the circuitry to music reward compared with inhibitory stimulation (cTBS), in which responses were blunted. Individual paired $t$ test comparisons within each ROI revealed that the maximum effect was located at the left caudate during the Pre-experience phase $\left(t_{(16)}=3.40\right.$, Bonferroni-corrected $p$ value, $\left.P_{\text {bonf }}<0.05\right)$. In addition, no significant changes were found when using a control ROI in the primary visual cortex $\left(F_{(1,16)}=0.51, p=0.49\right)$ nor at the left dlPFC $\left(F_{(1,16)}=0.05, p=0.95\right)$, which further 


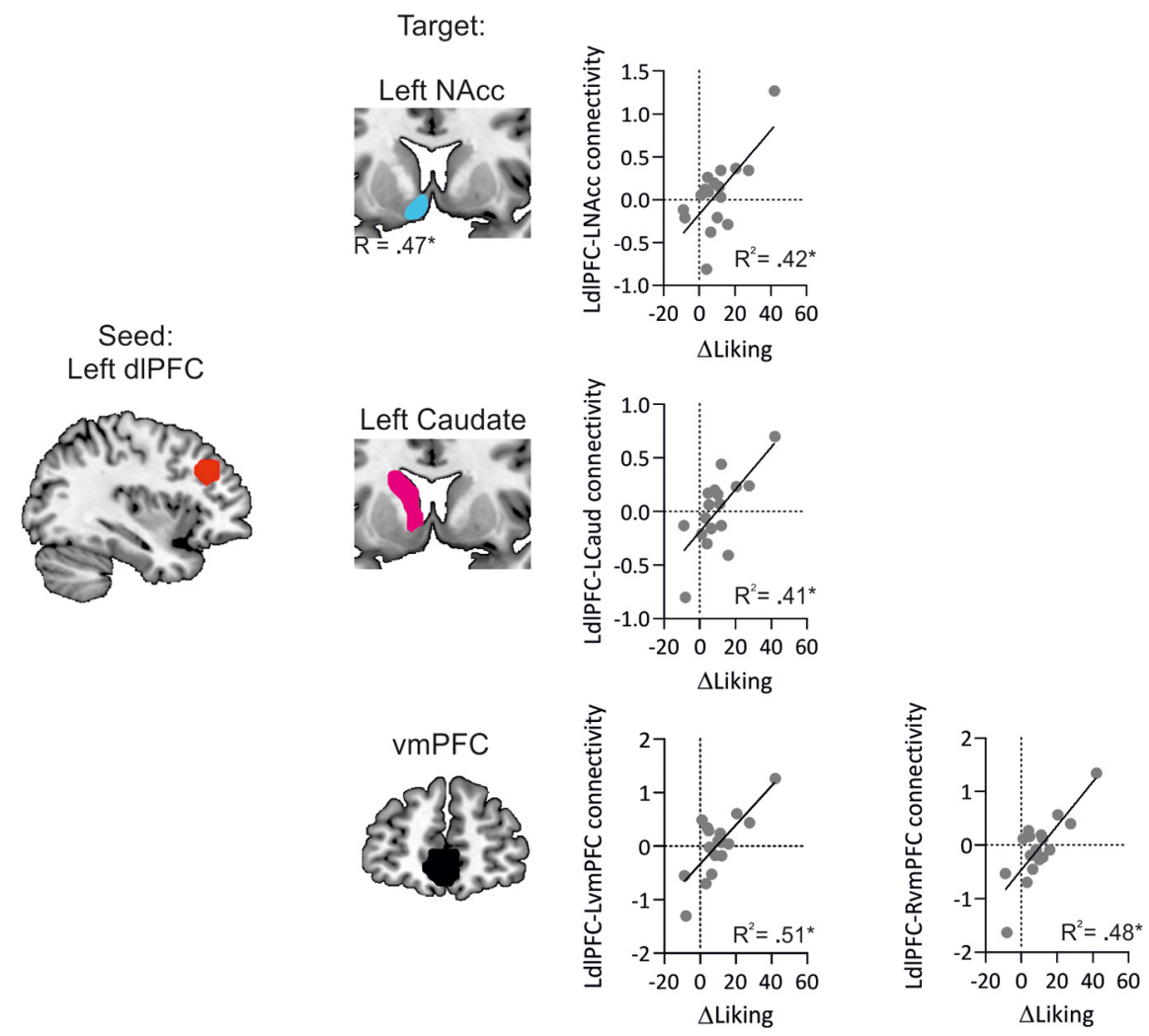

Figure 5. Functional connectivity using the left dIPFC as a seed. Scatter plots represent the significant relationships between individual differences in TMS modulation of subjective reports of pleasure and TMS-induced changes in the functional connectivity strength between the dIPFC and reward circuit in the Pre-experience phase.

supports the specificity of our results and excludes the possibility that changes in music reward sensitivity were driven by local changes in the target stimulated region.

Furthermore, we explored the relationship between TMSinduced changes in fMRI activity and TMS-induced changes in subjective reports of pleasure and motivation across participants. In order to assess this brain-behavior relationship, we performed robust regression analysis with individual TMS-induced changes (changes following iTBS changes following cTBS with respect to Sham) in subjective reports of pleasure ( $\Delta$ liking) and participants' bids ( $\Delta$ wanting), on the one hand; and subject-specific TMS-induced changes in reward-related activity in each ROI and reward phase, on the other $\left(\mathrm{SHV}_{\mathrm{iTBS}}-\mathrm{SHV}_{\mathrm{cTBS}}\right)$.

The analysis revealed that only TMS-induced changes in the bilateral NAcc $(\triangle \mathrm{NAcc})$, but not in the other ROIs, predicted individual differences in $\Delta$ liking and $\Delta$ wanting, although at distinct temporal phases (Fig. 4). TMS-induced changes in the NAcc during the Pre-experience phase predicted changes in the amount of money participants were willing to offer to purchase our music selection $\left(F_{(1,14)}=12.2, P_{\text {bonf }}<0.05, R^{2}=0.47\right.$, adjusted $R^{2}=0.43$ ), whereas changes in the same structure, but during the Experience phase, correlated with TMS-induced changes in subjective reports of pleasure $\left(F_{(1,15)}=11.7, P_{\text {bonf }}<\right.$ $0.05, R^{2}=0.44$, adjusted $\left.R^{2}=0.40\right)$. Notably, the relationship between $\Delta \mathrm{NAcc}$ and $\Delta$ wanting during the Pre-experience phase was greater than between $\Delta \mathrm{NAcc}$ and $\Delta$ wanting during the Experience phase $(Z=1.73, p=0.042)$ or between $\Delta$ NAcc and $\Delta$ liking during the Pre-experience phase $(Z=1.64, p=0.05)$. Similarly, the correlation between the $\triangle$ NAcc activity and $\Delta$ liking during the Experience phase was significantly greater than the correlation between the $\Delta \mathrm{NAcc}$ and $\Delta$ wanting during the Experience phase $(Z=1.72, p=0.042)$ and tended to be greater than between the $\triangle \mathrm{NAcc}$ and $\Delta$ liking during the Pre-experience phase $(Z=1.41, p=0.079)$. These findings support the idea of temporally dissociated correlations between the NAcc and both motivation and pleasure in musical reward.

As expected, no significant correlations were found between TMS-induced changes in the left dlPFC or the visual cortex and modulation of liking or wanting measures.

\section{Functional connectivity}

TMS-induced changes in the dopaminergic cortico-limbic pathway are thought to be driven by an effect on descending pathways from the left dlPFC to the striatum and the vmPFC. Based on that model, we wanted to investigate whether TMS-induced changes in the cross-talk between the left dlPFC and both the striatum and the vmPFC contributed to the modulation of musical reward sensitivity, even if there was no net change in the dlPFC activity induced by stimulation.

In order to assess the impact of TMS on the dlPFC connectivity, we performed a PPI analysis, which focused on enhanced interregional coupling as a function of hedonic value during the Pre-experience and the Experience phase, and after both excitatory and inhibitory stimulations compared with Sham (following a similar procedure to the previous fMRI analysis). We again focused on connectivity to the previously defined ROIs.

Individual differences in TMS-induced changes in subjective reports of pleasure ( $\Delta$ liking) were positively correlated with subject-specific changes in the connectivity strength between the left dlPFC (seed) and the (1) the left NAcc $\left(F_{(1,15)}=10.9, P_{\text {bonf }}\right.$ $<0.05, R^{2}=0.42$, adjusted $R^{2}=0.38$ ); (2) the left caudate $\left(F_{(1,15)}=10.5, P_{\text {bonf }}<0.05, R^{2}=0.41\right.$, adjusted $\left.R^{2}=0.37\right)$; and (3) the bilateral vmPFC (left: $F_{(1,15)}=15.7, P_{\text {bonf }}<0.05, R^{2}=$ 0.51 , adjusted $R^{2}=0.48$; right: $F_{(1,15)}=21.5, P_{\text {bonf }}<0.05, R^{2}=$ 0.59 , adjusted $R^{2}=0.56$ ) during the Pre-experience phase (Fig. 5). Thus, those individuals that reported the greatest difference of enjoyment between the excitatory and the inhibitory stimulation sessions were those individuals that showed the greatest changes in the connectivity strength between the target region (left dlPFC) and the reward circuitry during the Pre-experience and before the experience of pleasure.

Additionally, given the relevance of the cross-talk between the auditory cortex, particularly the right STG, and the reward circuitry, most notably the NAcc, in the experience of musical pleasure (Salimpoor et al., 2013; Martínez-Molina et al., 2016), we also performed an additional functional connectivity analysis using both the left and the right STG as seeds. In accord with the model, we found that the greater the TMSinduced changes in subjective reports of pleasure, the greater the TMS-induced changes in connectivity strength between the right STG and the right NAcc during the experience of pleasure $\left(F_{(1,15)}=9.92, P_{\text {bonf }}<0.05, R^{2}=0.40\right.$, adjusted $R^{2}=$ 

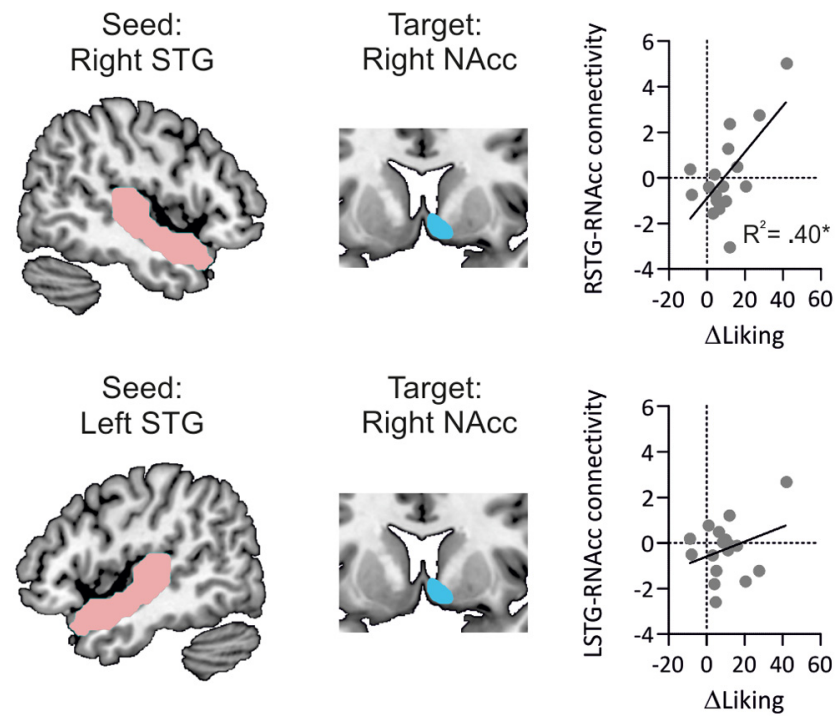

Figure 6. Functional connectivity using the right and left STG as a seed. Scatter plots represent the relationship between individual differences in TMS modulation of subjective reports of pleasure and TMS-induced changes in the functional connectivity strength between the right (top) and the left (bottom) STG and the NAcc in the Experience phase. Only changes in functional connectivity between the right STG and the right NAcc were associated with changes in subjective reports of pleasure. ${ }^{*} P_{\text {bonf }}<0.05$.

$0.36)$, but not during the Pre-experience $\left(F_{(1,15)}=3.54\right.$, $P_{\text {bonf }}>0.05, R^{2}=0.19$, adjusted $R^{2}=0.14$ ) (Fig. 6). These results further support the idea that functional interaction between cortical areas involved in auditory processing and reward-related structures is important for music-evoked pleasure. Finally, no significant correlations were found between $\Delta$ wanting or $\Delta$ liking and TMS-induced changes in the connectivity of the left dlPFC, the left STG, or the control ROI in the visual cortex.

\section{Discussion}

We investigated the temporal dynamics of striatal and vmPFC signals during the Pre-experience and Experience of musical reward, combining both TMS and fMRI to modulate and record brain activity. Previous studies have shown that TMS over the left dlPFC induces dopamine release and BOLD activations in the caudate (Strafella et al., 2001; Pogarell et al., 2006, 2007; Ko et al., 2008; Cho and Strafella, 2009; Hayashi et al., 2013; Dowdle et al., 2018). Separately, we have previously shown that this procedure effectively modulates music reward sensitivity at a behavioral and psychophysiological level (Mas-Herrero et al., 2018a). Importantly, here we replicated our previous behavioral findings in a new group of participants, reflecting the consistency and reproducibility of these effects. Concretely, excitatory stimulation of the fronto-striatal circuit increased both subjective reports of pleasure and motivation, whereas inhibition of this circuit led to the opposite effects in both. In addition, the TMS intervention also modulated the number and the duration of music-induced "chills." Because "chills" represent clear and discrete events, accompanied by changes in objective psychophysiological measures of emotional arousal, and are highly reproducible, they provide a reliable, objective indication of hedonic reactions to music (Sloboda, 1991; Grewe et al., 2009; Mas-Herrero et al., 2014; Laeng et al., 2016). These findings add significant evidence in favor of the interpretation that the reward circuitry modulation induces changes in affective reactions to music reward.
Interestingly, similar modulatory effects on both hedonic and motivational responses to music have been recently reported following the direct manipulation of systemic dopaminergic function via pharmacological action, thus complementing our findings, which provide anatomic specificity, by indicating neurochemical specificity (Ferreri et al., 2019). This pharmacological result and the fact that the implemented TMS procedure has previously been shown to induce striatal dopamine release suggest that the present findings could be mediated by changes in dopaminergic pathways. However, no direct measures of dopamine were taken in the current study.

Despite the consistency of TMS's behavioral outcomes in the current paradigm, the stimulation's precise mechanism in terms of the brain circuitry that may be modulated was previously not established. The present fMRI findings extend the behavioral results and clarify their neural basis by pointing to the NAcc as a relevant structure in the generation of music-induced reward. First, excitatory and inhibitory stimulation enhanced and disrupted, respectively, the responsiveness of striatal regions (including the NAcc) and the vmPFC to musical reward during both the Pre-experience and the Experience phases of the music reward cycle (Fig. 2). Second, TMS-induced changes in NAcc activations predicted modulations of both musical pleasure and motivation (Fig. 3). Third, greater TMS-induced changes in the connectivity strength between the left dlPFC and the NAcc were associated with greater positive changes in subjective reports of pleasure (Fig. 4). Thus, these results support the hypothesis that the engagement of the NAcc plays a causal role in music-induced reward.

Previous neuroimaging studies have consistently shown signal changes in the NAcc in response to musical pleasure across a large variety of experimental designs (Blood and Zatorre, 2001; Koelsch et al., 2006; Montag et al., 2011; Salimpoor et al., 2011, 2013; Koelsch, 2014; Mueller et al., 2015; Martínez-Molina et al., 2016; Shany et al., 2019; for a meta-analysis, see Mas-Herrero et al., 2021). Critically, a combined PET and fMRI study investigating the dynamics of dopaminergic signals in response to musicinduced chills showed that dopaminergic release and striatal engagement might occur at two different time points: before and after the experience of music-induced pleasure, with the former preferentially occurring in the caudate and the later associated with a dopaminergic release in the NAcc (Salimpoor et al., 2011). Despite its temporal dissociation, dopamine release in both structures correlated with subjective reports of pleasure, pointing to the relevance of both striatal regions in music-induced reward. Here, by stimulating the fronto-striatal circuitry formed by the left dlPFC-caudate via TMS, we extend these correlational findings, providing causal evidence that indirect stimulation of the striatum leads to modulation of musical reward sensitivity. Indeed, the main effect of the stimulation was located in the left caudate, consistent with previous studies showing dopaminergic release in this region following TMS over the left dlPFC (Strafella et al., 2001), and during the Pre-experience phase, following the temporal pattern previously identified by Salimpoor et al. (2011). However, TMS-induced changes in the left caudate did not appear to cause changes in pleasure or motivation directly, yet likely through caudate-NAcc interactions.

Anatomical, neurochemical, and brain lesion studies suggest that the NAcc is essential in motivational aspects of reward (Floresco, 2015; Berridge and Kringelbach, 2015). In particular, the NAcc, via dopaminergic transmission, is involved in the assignment of value/incentive salience to reward-predicting cues and relevant outcomes (Berridge and Robinson, 1998; Berridge 
and Kringelback, 2008; Schultz, 2016). These dopaminergic signals integrated into the NAcc are thought to guide decision-making, enhance approach or appetitive behavior, and fuel attention, learning, and memory (Berridge and Robinson, 1998; Redgrave et al., 1999; Ripollés et al., 2016, 2018; Schultz, 2016). Consequently, pharmacological manipulations of dopamine and intracranial stimulations in the NAcc increase anticipatory responses and participants' desire to obtain rewarding stimuli, such as food or drugs and enhance sexual arousal (Heath, 1972; Leyton et al., 2005; Evans et al., 2006). In line with this idea, we found that indirect modulation of the NAcc by means of prefrontal TMS stimulation led to changes in music reward-related responses. According to our fMRI findings, incentive/reward signals conveyed to the NAcc may concretely occur at two different time points, as previously identified by Salimpoor et al. (2011). First, before the experience of musical pleasure, this signal may reflect the expected value triggered by musical frames that generate expectations of potential pleasurable resolutions (e.g., through tension, verse-chorus forms, or chord progressions, among others) (Huron, 2006), leading to feelings of anticipation, which may progressively increase until the expected outcome is finally obtained.

Importantly, participants were exposed to their own favorite music and an experimenter-music selection that conformed to their musical preferences and listening habits (e.g., pop music) and, therefore, to a musical grammar they were familiar with and could generate predictions from it. In this regard, we and others have recently provided empirical evidence that musical pleasure often derives from evolving predictions, which derive themself from the music (Gold et al., 2019; Cheung et al., 2019). It is then very plausible that, even for novel music, the "Pre-experience" epochs represent, if not anticipation, then at least predictions, and that these are one component of musical pleasure. The pleasure increases related to predictions are not unexpected, even for novel music, as shown by the classic Wundt effect. Therefore, even for novel music, and even for music that does not induce chills, models do predict that before jumps in pleasure, there will have usually been a pleasurable anticipatory phase. Indeed, musical events that are completely surprising are unpleasant (Gold et al., 2019; Cheung et al., 2019).

Notably, TMS-induced changes in the engagement of the NAcc and before the experience of pleasure were associated with changes in the amount of money that participants were willing to pay. These findings further reinforce the idea that the NAcc's engagement during the Pre-experience phase reflects value computations. However, we acknowledge that the presence of behavioral ratings does not allow us to disentangle whether the value encoded during the Pre-experience phase reflected the value of the musical frame that was about to come [based on previous experience with the same musical piece (favorite music) or the same music style or genre (experimenter-selected music)] or the value of the action that participants were about to do. However, this question does not affect the current experiment's main conclusion, namely, that our TMS intervention modulated value computations and motivation signals in the NAcc while listening to music.

Next, a second signal is conveyed to the NAcc coinciding with the peak of pleasure, likely occurring when music-induced expectations are either violated or fulfilled. For instance, musical "chills" are often experienced following sudden dynamic changes triggered by unexpected harmonies or subtle changes of loudness (Sloboda, 1991; Panksepp, 1995; Guhn et al., 2007; Grewe et al., 2007; Nagel et al., 2008; Harrison and Loui, 2014), and songs that became popular (as measured by their ranking position in musical charts) show greater average surprise than those that did not (Miles et al., 2017). In accord with this idea, recent studies have shown that such music-elicited surprises may engage the NAcc as a function of predictability and value (Gold et al., 2019; Shany et al., 2019).

Musical expectations and surprises, formed via perceptual analysis taking place in the auditory cortex, as well as the frontal regions to which it connects (Petrides and Pandya, 2009; Bastos et al., 2012; Rohrmeier and Koelsch, 2012; Zatorre and Salimpoor, 2013; Albouy et al., 2015; Omigie et al., 2019), are likely to trigger the NAcc through functional and anatomic interactions of the latter with the STG (Zatorre, 2015). Previous neuroimaging studies have shown a cross-talk between these two structures while people listen to pleasant music, particularly in individuals with high sensitivity to musical reward (Salimpoor et al., 2013; Martínez-Molina et al., 2016; Freeman et al., 2018; Shany et al., 2019). In contrast, individuals with specific-musical anhedonia, who do not experience much pleasure from music (Mas-Herrero et al., 2014, 2018b), exhibit decreased functional and anatomic connections between the right STG and the NAcc (Martínez-Molina et al., 2016, 2019; Loui et al., 2017). Our results further support the relevance of this interaction. TMSinduced changes in musical pleasure were accompanied by changes in the functional connectivity between the right STG and the NAcc during the peak experience of musical pleasure. Notably, the effects were limited to the right, not the left STG, consistent with previous evidence showing dominant right lateralization in music processing (Johnsrude et al., 2000; Patterson et al., 2002; Schneider et al., 2005; Herholz et al., 2016).

In conclusion, current findings indicate that the engagement of cortico-striatal pathways is essential for the experience of musical reward. In addition, we provide further evidence that the reward circuitry treats music as any other reward/incentive salience signal, with its engagement coinciding with the anticipation and the experience of musical pleasure. Interestingly, our findings point to a dissociation between pre-experiential versus experiential components of music, and their role in the motivational and hedonic components of music reward, respectively. Finally, and more broadly, current findings also indicate that striatal pathways may be effectively targeted by noninvasive brain stimulation over cortical regions, highlighting the relevance of this procedure to better understand this circuitry's functioning.

\section{References}

Albouy P, Mattout J, Sanchez G, Tillmann B, Caclin A (2015) Altered retrieval of melodic information in congenital amusia: insights from dynamic causal modeling of MEG data. Front Hum Neurosci 9:20.

Bartra O, McGuire JT, Kable JW (2013) The valuation system: a coordinatebased meta-analysis of BOLD fMRI experiments examining neural correlates of subjective value. Neuroimage 76:412-427.

Bastos AM, Usrey WM, Adams RA, Mangun GR, Fries P, Friston KJ (2012) Canonical microcircuits for predictive coding. Neuron 76:695-711.

Berridge KC, Kringelbach ML (2008) Affective neuroscience of pleasure: reward in humans and animals. Psychopharmacology (Berl) 199:457480.

Berridge KC, Kringelbach ML (2015) Pleasure systems in the brain. Neuron 86:646-664.

Berridge KC, Robinson TE (1998) What is the role of dopamine in reward: hedonic impact, reward learning, or incentive salience? Brain Res Brain Res Rev 28:309-369.

Blood AJ, Zatorre RJ (2001) Intensely pleasurable responses to music correlate with activity in brain regions implicated in reward and emotion. Proc Natl Acad Sci USA 98:11818-11823. 
Brattico E, Bogert B, Alluri V, Tervaniemi M, Eerola T, Jacobsen T (2016) It's sad but I like it: the neural dissociation between musical emotions and liking in experts and laypersons. Front Hum Neurosci 9:676.

Bromberg-Martin ES, Matsumoto M, Hikosaka O (2010) Dopamine in motivational control: rewarding, aversive, and alerting. Neuron 68:815-834.

Chase HW, Kumar P, Eickhoff SB, Dombrovski AY (2015) Reinforcement learning models and their neural correlates: an activation likelihood estimation meta-analysis. Cogn Affect Behav Neurosci 15:435-459.

Cheung VK, Harrison PM, Meyer L, Pearce MT, Haynes JD, Koelsch S (2019) Uncertainty and surprise jointly predict musical pleasure and amygdala, hippocampus, and auditory cortex activity. Current Biologyi 29:4084-4092.

Cho SS, Strafella AP (2009) RTMS of the left dorsolateral prefrontal cortex modulates dopamine release in the ipsilateral anterior cingulate cortex and orbitofrontal cortex. PLoS One 4:e6725.

Diekhof EK, Kaps L, Falkai P, Gruber O (2012) The role of the human ventral striatum and the medial orbitofrontal cortex in the representation of reward magnitude: an activation likelihood estimation meta-analysis of neuroimaging studies of passive reward expectancy and outcome processing. Neuropsychologia 50:1252-1266.

Dowdle LT, Brown TR, George MS, Hanlon CA (2018) Single pulse TMS to the dlPFC, compared to a matched Sham control, induces a direct, causal increase in caudate, cingulate, and thalamic BOLD signal. Brain Stimul 11:789-796.

Evans AH, Pavese N, Lawrence AD, Tai YF, Appel S, Doder M, Brooks DJ, Lees AJ, Piccini P (2006) Compulsive drug use linked to sensitized ventral striatal dopamine transmission. Ann Neurol 59:852-858.

Ferreri L, Mas-Herrero E, Zatorre RJ, Ripollés P, Gomez-Andres A, Alicart H, Olivé G, Marco-Pallarés J, Antonijoan RM, Valle M, Riba J, Rodriguez-Fornells A (2019) Dopamine modulates the reward experiences elicited by music. Proc Natl Acad Sci USA 116:3793-3798.

Floresco SB (2015) The nucleus accumbens: an interface between cognition, emotion, and action. Annu Rev Psychol 66:25-52.

Freeman TP, Pope RA, Wall MB, Bisby JA, Luijten M, Hindocha C, Mokrysz C, Lawn W, Moss A, Bloomfield MA, Morgan CJ, Nutt DJ, Curran HV (2018) Cannabis dampens the effects of music in brain regions sensitive to reward and emotion. Int J Neuropsychopharmacol 21:21-32.

Friston KJ, Buechel C, Fink GR, Morris J, Rolls E, Dolan RJ (1997) Psychophysiological and modulatory interactions in neuroimaging. NeuroImage 6:218-229.

Gold BP, Mas-Herrero E, Zeighami Y, Benovoy M, Dagher A, Zatorre RJ (2019) Musical reward prediction errors engage the nucleus accumbens and motivate learning. Proc Natl Acad Sci USA 116:3310-3315.

Grewe O, Nagel F, Kopiez R, Altenmüller E (2005) How does music arouse "chills?" Investigating strong emotions, combining psychological, physiological, and psychoacoustical methods. Ann NY Acad Sci 1060:446-449.

Grewe O, Nagel F, Kopiez R, Altenmüller E (2007) Emotions over time: synchronicity and development of subjective, physiological, and facial affective reactions to music. Emotion 7:774-788.

Grewe O, Kopiez R, Altenmüller E (2009) Chills as an indicator of individual emotional peaks. Ann NY Acad Sci 1169:351-354.

Guhn M, Hamm A, Zentner M (2007) Physiological and musico-acoustic correlates of the chill response. Music Perception 24:473-484.

Hammers A, Allom R, Koepp MJ, Free SL, Myers R, Lemieux L, Mitchell TN, Brooks DJ, Duncan JS (2003) Three-dimensional maximum probability atlas of the human brain, with particular reference to the temporal lobe. Hum Brain Mapp 19:224-247.

Harrison L, Loui P (2014) Thrills, chills, frissons, and skin orgasms: toward an integrative model of transcendent psychophysiological experiences in music. Front Psychol 5:790.

Hayashi T, Ko JH, Strafella AP, Dagher A (2013) Dorsolateral prefrontal and orbitofrontal cortex interactions during self-control of cigarette craving. Proc Natl Acad Sci USA 110:4422-4427.

Heath RG (1972) Pleasure and brain activity in man: deep and surface electroencephalograms during orgasm. J Nerv Ment Dis 154:3-18.

Herholz SC, Coffey EB, Pantev C, Zatorre RJ (2016) Dissociation of neural networks for predisposition and for training-related plasticity in auditory-motor learning. Cereb Cortex 26:3125-3134.

Huron DB (2006) Sweet anticipation: music and the psychology of expectation. Cambridge, MA: Massachusetts Institute of Technology.
Johnsrude IS, Penhune VB, Zatorre RJ (2000) Functional specificity in the right human auditory cortex for perceiving pitch direction. Brain 123: 155-163.

Ko JH, Monchi O, Ptito A, Bloomfield P, Houle S, Strafella AP (2008) Theta burst stimulation-induced inhibition of dorsolateral prefrontal cortex reveals hemispheric asymmetry in striatal dopamine release during a setshifting task: a TMS-[(11)C]raclopride PET study. Eur J Neurosci 28:2147-2155

Koelsch S (2014) Brain correlates of music-evoked emotions. Nat Rev Neurosci 15:170-180.

Koelsch S, Fritz T, Cramon DY, Müller K, Friederici AD (2006) Investigating emotion with music: an fMRI study. Hum Brain Mapp 27:239-250.

Laeng B, Eidet LM, Sulutvedt U, Panksepp J (2016) Music chills: the eye pupil as a mirror to music's soul. Conscious Cogn 44:161-178.

Leyton M, Casey KF, Delaney JS, Kolivakis T, Benkelfat C (2005) Cocaine craving, euphoria, and self-administration: a preliminary study of the effect of catecholamine precursor depletion. Behav Neurosci 119:16191627.

Loui P, Patterson S, Sachs ME, Leung Y, Zeng T, Przysinda E (2017) White matter correlates of musical anhedonia: implications for evolution of music. Front Psychol 8:1664.

Luijten M, Schellekens AF, Kühn S, Machielse MW, Sescousse G (2017) Disruption of reward processing in addiction: an image-based meta-analysis of functional magnetic resonance imaging studies. JAMA Psychiatry 74:387-398.

Martínez-Molina N, Mas-Herrero E, Rodríguez-Fornells A, Zatorre RJ, Marco-Pallarés J (2016) Neural correlates of specific musical anhedonia. Proc Natl Acad Sci USA 113:E7337-E7345.

Martínez-Molina N, Mas-Herrero E, Rodríguez-Fornells A, Zatorre RJ, Marco-Pallarés J (2019) White matter microstructure reflects individual differences in music reward sensitivity. J Neurosci 39:5018-5027.

Mas-Herrero E, Zatorre RJ, Rodriguez-Fornells A, Marco-Pallarés J (2014) Dissociation between musical and monetary reward responses in specific musical anhedonia. Curr Biol 24:699-704.

Mas-Herrero E, Dagher A, Zatorre RJ (2018a) Modulating musical reward sensitivity up and down with transcranial magnetic stimulation. Nat Hum Behav 2:27-32.

Mas-Herrero E, Karhulahti M, Marco-Pallares J, Zatorre RJ, RodriguezFornells A (2018b) The impact of visual art and emotional sounds in specific musical anhedonia. Prog Brain Res 237:399-413.

Mas-Herrero E, Sescousse G, Cools R, Marco-Pallarés J (2019) The contribution of striatal pseudo-reward prediction errors to value-based decisionmaking. Neuroimage 193:67-74.

Mas-Herrero E, Maini L, Sescousse G, Zatorre RJ (2021) Common and distinct neural correlates of music and food-induced pleasure: a coordinatebased meta-analysis of neuroimaging studies. Neurosci Biobehav Rev 123:61-71.

Meyer LB (1956) Emotion and meaning in music. Chicago: Chicago UP.

Miles SA, Rosen DS, Grzywacz NM (2017) A statistical analysis of the relationship between harmonic surprise and preference in popular music. Front Hum Neurosci 11:263.

Montag C, Reuter M, Axmacher N (2011) How one's favorite song activates the reward circuitry of the brain: personality matters! Behav Brain Res 225:511-514.

Mueller K, Fritz T, Mildner T, Richter M, Schulze K, Lepsien J, Schroeter ML, Möller HE (2015) Investigating the dynamics of the brain response to music: a central role of the ventral striatum/nucleus accumbens. Neuroimage 116:68-79.

Nagel F, Kopiez R, Grewe O, Altenmüller E (2008) Psychoacoustical correlates of musically induced chills. Musicae Scientiae 12:101-113.

Oldham S, Murawski C, Fornito A, Youssef G, Yücel M, Lorenzetti V (2018) The anticipation and outcome phases of reward and loss processing: a neuroimaging meta-analysis of the monetary incentive delay task. Hum Brain Mapp 39:3398-3418.

Omigie D, Pearce M, Lehongre K, Hasboun D, Navarro V, Adam C, Samson $S$ (2019) Intracranial recordings and computational modeling of music reveal the time course of prediction error signaling in frontal and temporal cortices. J Cogn Neurosci 31:855-873.

Panksepp J (1995) The emotional sources of "chills" induced by music. Music Perception 13:171-207. 
Patterson RD, Uppenkamp S, Johnsrude IS, Griffiths TD (2002) The processing of temporal pitch and melody information in auditory cortex. Neuron 36:767-776.

Petrides M, Pandya DN (2009) Distinct parietal and temporal pathways to the homologues of Broca's area in the monkey. PLoS Biol 7:e1000170.

Pogarell O, Koch W, Pöpperl G, Tatsch K, Jakob F, Zwanzger P, Mulert C, Rupprecht R, Möller HJ, Hegerl U, Padberg F (2006) Striatal dopamine release after prefrontal repetitive transcranial magnetic stimulation in major depression: preliminary results of a dynamic $\left[{ }^{123} \mathrm{I}\right]$ IBZM SPECT study. J Psychiatr Res 40:307-314.

Pogarell O, Koch W, Pöpperl G, Tatsch K, Jakob F, Mulert C, Grossheinrich N, Rupprecht R, Möller HJ, Hegerl U, Padberg F (2007) Acute prefrontal rTMS increases striatal dopamine to a similar degree as D-amphetamine. Psychiatry Res 156:251-255.

Redgrave P, Prescott TJ, Gurney K (1999) Is the short-latency dopamine response too short to signal reward error? Trends Neurosci 22:146-151.

Ripollés P, Marco-Pallarés J, Alicart H, Tempelmann C, Rodríguez-Fornells A, Noesselt T (2016) Intrinsic monitoring of learning success facilitates memory encoding via the activation of the SN/VTA-hippocampal loop. Elife 5:e17441.

Ripollés P, Ferreri L, Mas-Herrero E, Alicart H, Gómez-Andrés A, MarcoPallares J, Antonijoan RM, Noesselt T, Valle M, Riba J, RodriguezFornells A (2018) Intrinsically regulated learning is modulated by synaptic dopamine signaling. Elife 7:e38113.

Rohrmeier MA, Koelsch S (2012) Predictive information processing in music cognition: a critical review. Int J Psychophysiol 83:164-175.

Salimpoor VN, Benovoy M, Longo G, Cooperstock JR, Zatorre RJ (2009) The rewarding aspects of music listening are related to degree of emotional arousal. PLoS One 4:e7487.

Salimpoor VN, Benovoy M, Larcher K, Dagher A, Zatorre RJ (2011) Anatomically distinct dopamine release during anticipation and experience of peak emotion to music. Nat Neurosci 14:257-262.

Salimpoor VN, van den Bosch I, Kovacevic N, McIntosh AR, Dagher A, Zatorre RJ (2013) Interactions between the nucleus accumbens and auditory cortices predict music reward value. Science 340:216-219.
Schneider P, Sluming V, Roberts N, Scherg M, Goebel R, Specht HJ, Dosch HG, Bleeck S, Stippich C, Rupp A (2005) Structural and functional asymmetry of lateral Heschl's gyrus reflects pitch perception preference. Nat Neurosci 8:1241-1247.

Schultz W (2016) Dopamine reward prediction-error signalling: a two-component response. Nat Rev Neurosci 17:183-195.

Schultz W, Dayan P, Montague PR (1997) A neural substrate of prediction and reward. Science 275:1593-1599.

Sescousse G, Caldú X, Segura B, Dreher JC (2013) Processing of primary and secondary rewards: a quantitative meta-analysis and review of human functional neuroimaging studies. Neurosci Biobehav Rev 37:681-696.

Shany O, Singer N, Gold BP, Jacoby N, Tarrasch R, Hendler T, Granot R (2019) Surprise-related activation in the nucleus accumbens interacts with music-induced pleasantness. Soc Cogn Affect Neurosci 14:459-470.

Sloboda JA (1991) Music structure and emotional response: some empirical findings. Psychol Music 19:110-120.

Steiger JH (1980) Testing pattern hypotheses on correlation matrices: alternative statistics and some empirical results. Multivariate Behav Res 15:335352.

Strafella AP, Paus T, Barrett J, Dagher A (2001) Repetitive transcranial magnetic stimulation of the human prefrontal cortex induces dopamine release in the caudate nucleus. J Neurosci 21:RC157.

Wassiliwizky E, Koelsch S, Wagner V, Jacobsen T, Menninghaus W (2017) The emotional power of poetry: neural circuitry, psychophysiology and compositional principles. Soc Cogn Affect Neurosci 12:1229-1240.

Weiskopf N, Hutton C, Josephs O, Deichmann R (2006) Optimal EPI parameters for reduction of susceptibility-induced BOLD sensitivity losses: a whole-brain analysis at $3 \mathrm{~T}$ and $1.5 \mathrm{~T}$. Neuroimage 33:493-504

Zatorre RJ (2015) Musical pleasure and reward: mechanisms and dysfunction. Ann NY Acad Sci 1337:202-211.

Zatorre RJ, Salimpoor VN (2013) From perception to pleasure: music and its neural substrates. Proc Natl Acad Sci USA 110 Suppl 2:10430-10437. 